\title{
ON THE FOURIER EXPANSIONS OF JACOBI FORMS
}

\author{
HOWARD SKOGMAN
}

Received 19 January 2004

\begin{abstract}
We use the relationship between Jacobi forms and vector-valued modular forms to study the Fourier expansions of Jacobi forms of indexes $p, p^{2}$, and $p q$ for distinct odd primes $p, q$. Specifically, we show that for such indexes, a Jacobi form is uniquely determined by one of the associated components of the vector-valued modular form. However, in the case of indexes of the form $p q$ or $p^{2}$, there are restrictions on which of the components will uniquely determine the form. Moreover, for indexes of the form $p$, this note gives an explicit reconstruction of the entire Jacobi form from a single associated vector-valued modular form component. That is, we show how to start with a single associated vector component and use specific matrices from $\mathrm{Sl}_{2}(\mathbb{Z})$ to find the other components and hence the entire Jacobi form. These results are used to discuss the possible modular forms of half-integral weight associated to the Jacobi form for different subgroups.
\end{abstract}

2000 Mathematics Subject Classification: 11F50, 11F30.

1. Introduction. Jacobi forms of weight $k$ and index $m$ over the rational numbers possess a Fourier expansion of the form

$$
\sum_{n=0}^{\infty} \sum_{\left\{r \in \mathbb{Z} \mid 4 n m-r^{2} \geq 0\right\}} c(n, r) q^{n} \xi^{r}, \quad q=e^{2 \pi i \tau}, \xi=e^{2 \pi i z}
$$

An easy calculation as in Eichler and Zagier's book [3] based on the invariance with respect to $\mathbb{Z}^{2}$ yields that $c(n, r)$ depends only on $r$ modulo $2 m$ and on $4 n m-r^{2}$. Thus the Fourier coefficients break into congruence classes modulo $2 m$ and this is the basis of the connection to vector-valued modular forms. The vector-valued modular forms have a prescribed transformation matrix with respect to the inversion in $\mathrm{Sl}_{2}(\mathbb{Z})$ and this puts restrictions on the possible Fourier expansions of a Jacobi form. Specifically, it forces a lower bound on the number of congruence classes of $r \bmod 2 m$ that have nonzero coefficients. In this note, we explore this lower bound and calculate the relation to vector-valued modular forms in the case of congruence subgroups. This leads to new isomorphisms between vector-valued forms of higher level and Jacobi forms on subgroups of the Jacobi group. In particular, the transformation of the vector-valued modular form gives the information to generate a Jacobi form from a congruence class of its Fourier coefficients. This construction is given explicitly in this work using a special set of matrices. There are related results in Skoruppa's thesis [9] although the methods used here are completely different (and very elementary) and involve only the Fourier expansions, the transformation formulas, and evaluation of Gauss sums. 
However, in [9], there are far more maps from Jacobi forms to half-integral weight modular forms.

We will use the notation $e^{m}(\cdot)=e^{2 \pi m i(\cdot)}, e_{m}(\cdot)=e^{2 \pi i(\cdot) / m},(a, b)=\operatorname{gcd}(a, b)$, and $\varphi(n)$ is the Euler phi-function. For convenience, representatives of congruence classes modulo $2 m$ (where $m$ is a rational integer) will be taken from the usual set $0,1,2, \ldots$, $2 m-1$.

2. Jacobi forms. (All of the material in this section can be found in [3].) Jacobi forms are functions satisfying certain transformation formulas under the group $\Gamma^{J}=\Gamma^{J}(\mathbb{Z})=$ $\mathrm{Sl}_{2}(\mathbb{Z}) \ltimes \mathbb{Z}^{2}$. The definition given in [3] is as follows.

DEFINITION 2.1. A Jacobi form of weight $k \in \mathbb{Z}^{+}$and index $m \in \mathbb{Z}^{+}$is a function $f(\tau, z): \mathfrak{h} \times \mathbb{C} \rightarrow \mathbb{C}$ that is analytic in both variables and satisfies

(1) $f((a \tau+b) /(c \tau+d), z /(c \tau+d))=(c \tau+d)^{k} e^{m}\left(c z^{2} /(c \tau+d)\right) f(\tau, z)$ for all $\left(\begin{array}{ll}a & b \\ c & d\end{array}\right) \in \mathrm{Sl}_{2}(\mathbb{Z})$

(2) $f(\tau, z+\lambda \tau+\mu)=e^{m}\left(-\lambda^{2} \tau-2 \lambda z\right) f(\tau, z)$ for all $[\lambda, \mu] \in \mathbb{Z}^{2}$.

Jacobi forms have a Fourier expansion of the form (1.1), where the condition on the second sum is a condition to make the function analytic as $\tau \rightarrow i \infty$. The second transformation law forces $c(n, r)=c\left(n^{\prime}, r^{\prime}\right)$ if $r \equiv r^{\prime} \bmod 2 m$ and $4 n m-r^{2}=4 n^{\prime} m-$ $\left(r^{\prime}\right)^{2}$ and we write $c(n, r)=c_{\mu}\left(N=4 n m-r^{2}\right)$, where $r \equiv \mu \bmod 2 m$. This allows a Jacobi form to be written as a linear combination of fixed theta functions. For each congruence class modulo $2 m$, define

$$
\theta_{\mu, m}(\tau, z)=\sum_{n \in \mathbb{Z}, n \equiv \mu \bmod 2 m} q^{n^{2} / 4 m} \xi^{n}, \quad h_{\mu}(\tau)=\sum_{N=0}^{\infty} c_{\mu}(N) q^{N / 4 m},
$$

where the coefficients $c_{\mu}(N)$ are defined to be zero unless $N \equiv-\mu^{2} \bmod 2 m$. With this notation, we have

$$
f(\tau, z)=\sum_{\mu \bmod 2 m} h_{\mu}(\tau) \theta_{\mu, m}(\tau, z)
$$

This decomposition leads to the theorem.

THEOREM 2.2 [3, Theorem 5.1]. Equation (2.2) gives an isomorphism between Jacobi forms of weight $k$ and index $m$ on $\Gamma^{J}$ and vector-valued modular forms $\left(h_{\mu}(\tau)\right)_{\mu \bmod 2 m}$ satisfying

(i) $h_{\mu}(\tau+1)=e_{4 m}\left(-\mu^{2}\right) h_{\mu}(\tau)$,

(ii) $h_{\mu}(-1 / \tau)=\left(\tau^{k} / \sqrt{2 m \tau / i}\right) \sum_{\nu \bmod 2 m} e_{2 m}(\mu \nu) h_{v}(\tau)$, and bounded as $\operatorname{Im}(\tau) \rightarrow \infty$.

This theorem gives relationships among the $h_{\mu}(\tau)$. If one $h_{\mu}(\tau)=0$ (i.e., is identically zero), then there is a linear dependence equation among the other $h_{v}(\tau)$ arising from the inversion formula. As an example, there are no Jacobi forms on $\Gamma^{J}$ with only one $h_{\mu}(\tau) \neq 0$ regardless of weight and index. The question arises as to how many of the $h_{\mu}(\tau)$ must be nonzero and how restrictive these conditions are on the Fourier expansion of the Jacobi form. 
3. Conditions on the Fourier expansions. The first basic restriction on the Fourier expansion of $f(\tau, z)$ is that since $\left(\begin{array}{cc}-1 & 0 \\ 0 & -1\end{array}\right)$ is in $\mathrm{Sl}_{2}(\mathbb{Z})$, we have that $f(\tau,-z)=(-1)^{k} f(\tau$, $z$ ) and therefore $h_{\mu}(\tau)=(-1)^{k} h_{-\mu}(\tau)$. From this, it is clear that for odd weights, $h_{0}(\tau)=h_{m}(\tau)=0$. This implies that there are no theta functions of odd weight for the full Jacobi group since the theta functions always have a nonzero $h_{0}(\tau)$ component. (This also follows from the basic property that 2 divides the level of any quadratic form unless 8 divides the rank.)

At this point, it is already possible to create some interesting information of which the following is simply an example.

EXAMPLE 3.1. Consider a Jacobi form $f(\tau, z)$ of odd weight and index 2. Such a form will only have two associated nonzero vector components $h_{1}(\tau)$ and $h_{3}(\tau)$. In addition, we know that $h_{1}(\tau)=(-1)^{k} h_{3}(\tau)$ and so

$$
h_{1}\left(\frac{-1}{\tau}\right)=\frac{\tau^{k}}{\sqrt{i \tau}} h_{1}(\tau)
$$

Therefore, the space of Jacobi forms of odd weight and index 2 is isomorphic to the space of modular forms $h_{1}(\tau)$ of weight $k-1 / 2$ satisfying the above inversion and $h_{1}(\tau+1)=e^{\pi i / 4} h_{1}(\tau)$.

This relationship to vector-valued modular forms is actually very restrictive on the possible Fourier expansions of Jacobi forms.

THEOREM 3.2. If $f(\tau, z)$ is a Jacobi form of index $m$, an odd prime, then all of the corresponding $h_{f, \mu}(\tau)$ are nonzero excluding $\mu=0, m$ if the weight is odd.

Proof. The argument is based on the transformation formula for the vector-valued modular form. Note that in the case $m$ is prime, there are only two elements $( \pm \mu)$ in each square class modulo $2 m$ (with just one element in the 0 and $m$ classes). To begin, assume $h_{\mu}(\tau)=0$ for $\mu$ other than $0, m$ (independent of weight); then $h_{-\mu}(\tau)=0$ by the relation $h_{\mu}(\tau)=(-1)^{k} h_{-\mu}(\tau)$. If $h_{\mu}(\tau)=0$, then $h_{\mu}(-1 / \tau)=C \sum_{\nu \bmod 2 m} e_{2 m}(\mu \nu) h_{\nu}(\tau)$ $=0$, where $C$ is the term involving $\tau^{k}$. By examining the expansion of each of the $h_{v}(\tau)$, only $h_{v}(\tau)$ and $h_{-v}(\tau)$ have terms of the form $q^{-v^{2} / 4 m} q^{n}$ (this is true even if $m=2$ ), and using these two inversion equations, we have $e_{2 m}(\mu \nu) h_{v}(\tau)+e_{2 m}(-\mu \nu) h_{-v}(\tau)=$ 0 and $e_{2 m}(-\mu \nu) h_{\nu}(\tau)+e_{2 m}(\mu \nu) h_{-v}(\tau)=0$ (from $h_{-\mu}=0$ ), and therefore $h_{v}(\tau)=$ $e_{m}(-\mu \nu) h_{-v}(\tau)$ and $h_{v}(\tau)=e_{m}(\mu \nu) h_{-v}(\tau)$. But unless $m \mid \mu \nu, \mu \nu$ and $-\mu \nu$ are not equivalent modulo $m$ (unless $m=2$ ). In the odd weight case, $h_{0}(\tau)=h_{m}(\tau)=0$, therefore $h_{v}(\tau)=0$ for all $v \bmod 2 m$. In the even weight case, $h_{0}(\tau)=0=h_{m}(\tau)$ because they are the only elements of their square classes (unless $m=2$ ) and the inversion formula for $h_{\mu}(\tau)$ implies they are zero.

In the even weight case, if $h_{0}(\tau)=0$, then $h_{\mu}(\tau)=0$ for all of the other $\mu \bmod 2 m$ $(\mu \neq m)$ since $h_{0}(-1 / \tau)=0$ implies $h_{\mu}(\tau)+h_{-\mu}(\tau)=0$. However, $h_{\mu}(\tau)=(-1)^{k} h_{-\mu}(\tau)$ and therefore $h_{\mu}(\tau)=0$ and $h_{m}(\tau)$ is zero again because it is the only element in its square class. A similar argument works if we assume that $h_{m}(\tau)$ is zero instead of $h_{0}(\tau)$. 
This proposition gives an interesting corollary in the case of prime index that any component (other than the 0th or $m$ th for odd weight) of a vector-valued modular form satisfying the conditions in (2.2) entirely determines the form. Or equivalently this may be stated as follows.

COROLLARY 3.3. If $f(\tau, z)=\sum_{n, r} c(n, r) q^{n} \xi^{r}$ is a Jacobi form of weight $k$ and odd prime index $m$, then $f$ is entirely determined by any congruence class $r \equiv \mu \bmod 2 m$ (i.e., $h_{\mu}(\tau)$ from (2.2)) of its Fourier coefficients (where $\mu \neq 0, m$ if the weight $k$ is odd).

Proof. Assume that two Jacobi forms $f_{1}(\tau, z), f_{2}(\tau, z)$ of the same weight and odd prime index have the same associated vector component. Then the difference $f_{1}(\tau, z)-f_{2}(\tau, z)$ is a Jacobi form of the same weight and index with one associated vector component identically zero. Hence the form is identically zero and $f_{1}(\tau, z)=$ $f_{2}(\tau, z)$.

Next consider the case of two prime factors in the index. Note that by the Chinese remainder theorem, if $m=p q$ for distinct primes $p, q$, then the structure of the square classes is as follows. There are $\varphi(p q) / 4$ squares with four square roots (those with $(\mu, p q)=1)$, there are $\varphi(p) / 2+\varphi(q) / 2$ squares with two square roots (those with $(\mu, p q)=p$ or $q$ ), and there are $0, m$ which have one square root each (recall $\varphi(n)$ is the Euler phi-function).

THEOREM 3.4. If $f(\tau, z)$ is a Jacobi form of index $m=p q$ for odd primes $p, q$ and the associated $h_{v}(\tau)=0$ for some $v \bmod 2 p q$ with $(\nu, p q)=1$, then $f$ is identically zero. If the weight is even and $h_{v}(\tau)=0$ for some $v$ with $(v, p q)>1$, then all of the components $h_{\mu}(\tau)$ for $(\mu, p q)>1$ are zero. If the weight is odd and $h_{\nu}(\tau)=0$ for a $v$ with $(\nu, p q)=p$ (or q), then all of the $h_{\mu}(\tau)$ with $(\mu, p q)=p$ (resp., q) are zero.

Proof. We begin with the second part of the proposition. If $h_{\mu}(\tau)=0$ for $p \mid \mu$ (or $q \mid \mu)$ and $\mu \neq 0, m$, then $h_{-\mu}(\tau)=0$ as well. Then for any $h_{a p}(\tau)(1 \leq a<q)$, there are two formulas relating $h_{a p}(\tau)$ and $h_{-a p}(\tau)$ that lead to $h_{a p}(\tau)=e_{2 p q}(2 a p \mu) h_{-a p}(\tau)$ and $h_{a p}(\tau)=e_{2 p q}(-2 a p \mu) h_{-a p}(\tau)$ which implies $h_{a p}(\tau)=0$ because $2 p q$ does not divide $2 a p \mu$ (since $(a p \mu, q)=1)$. Similarly this argument can be used to show the same result with $q$ replacing $p$. Now $h_{0}(\tau), h_{p q}(\tau)$ are both zero (for both even and odd weights) because for odd primes $p, q$, they are the only elements in their respective square classes (modulo $2 p q$ ). Then for even weights, all of the $h_{v}(\tau)=0$ for $q \mid v$ (or $p \mid v)$ because $h_{v}(\tau)=(-1)^{k} h_{-v}(\tau)$ and the inversion for $h_{0}$ leads to $h_{v}(\tau)=-h_{-v}(\tau)$. Note that if it is first assumed $h_{0}(\tau)$ or $h_{m}(\tau)$ is zero (and the weight is even), then use $h_{v}(\tau)=(-1)^{k} h_{-v}(\tau)$ as the second relation.

Now assume $h_{v}(\tau)=0$, where $(\nu, m)=1$. Then $h_{-v}(\tau)=0$ also. There are now two other congruence classes modulo $2 m$ called $\mu,-\mu$ such that $\mu^{2} \equiv v^{2} \bmod 2 m$. Because $h_{\nu}(\tau)$ and $h_{-v}(\tau)$ are zero, their inversion formulas give $h_{\mu}(\tau)=e_{2 m}(2 \mu \nu) h_{-\mu}(\tau)$ and $h_{\mu}(\tau)=e_{2 m}(-2 \mu \nu) h_{-\mu}(\tau)$ which is impossible because $(\mu \nu, m)=1$. These relations force $h_{\sigma}(\tau)=0$ for all $\sigma$ such that $(\sigma, p q)>1$ because each of these $\sigma$ has (at most) two elements in its square class and four nontrivial relations among the two functions. To complete the argument, take another square class $a^{2} \bmod 2 m$ such that $(a, p q)=1$. This class will have four square roots; call them $a,-a, b,-b$. Since $h_{ \pm v}(\tau)=h_{ \pm \mu}(\tau)=0$, 
there are four equations in the $h_{ \pm a}, h_{ \pm b}$ which can be written as

$$
\left(\begin{array}{cccc}
e_{2 m}(v a) & e_{2 m}(-v a) & e_{2 m}(\nu b) & e_{2 m}(-v b) \\
e_{2 m}(-v a) & e_{2 m}(v a) & e_{2 m}(-v b) & e_{2 m}(\nu b) \\
e_{2 m}(\mu a) & e_{2 m}(-\mu a) & e_{2 m}(\mu b) & e_{2 m}(-\mu b) \\
e_{2 m}(-\mu a) & e_{2 m}(\mu a) & e_{2 m}(-\mu b) & e_{2 m}(\mu b)
\end{array}\right)\left(\begin{array}{c}
h_{a}(\tau) \\
h_{-a}(\tau) \\
h_{b}(\tau) \\
h_{-b}(\tau)
\end{array}\right)=\left(\begin{array}{l}
0 \\
0 \\
0 \\
0
\end{array}\right)
$$

This matrix is nonsingular which can be shown by direct computation, and therefore all of the $h_{ \pm a}(\tau), h_{ \pm b}(\tau)$ are zero.

Again this proposition can be interpreted to say that a Jacobi form of index $p q$ is entirely determined by any of the corresponding $h_{\mu}(\tau)$ for $(\mu, 2 p q)=1$. Similar analysis can be used on general indexes. However, as the number of prime factors increases, the square classes become more complicated.

Given these two propositions, one might guess that, in general, the component functions $h_{\mu}(\tau)$, with $\mu$, relatively prime to $m$, cannot be zero. However, when the index $m=p^{2}$ for an odd prime $p$, the situation is reversed. For this case, the associated $h_{\mu}(\tau)$ with $(\mu, p)=1$ may all be zero, whereas if one of the (nontrivial) $h_{a p}(\tau)$ is zero, then the Jacobi form must be zero.

THEOREM 3.5. If $f(\tau, z)$ is a Jacobi form of odd weight and index $m=p^{2}$ for $p$ an odd prime, then if the associated $h_{\mu}(\tau)=0$ for any $\mu\left(\mu \neq 0, p^{2}\right)$, then $h_{v}(\tau)=0$ for all $\nu \bmod 2 p^{2}$ (i.e., $f$ is identically zero). If the weight is even and $h_{\mu}(\tau)=0$ for any $\mu$, then $h_{\nu}(\tau)=0$ for all $(\nu, p)=1$, and if $p \mid \mu$, then $f(\tau, z)$ is identically zero.

Proof. The difference here is the structure of the square classes. In general, $a^{2}$ has only two square roots $( \pm a)$ if $(p, a)=1$ but there are $p$ elements such that $b^{2} \equiv$ $0 \bmod 2 p^{2}$ (there are also $p$ such that $b^{2} \equiv p^{2} \bmod 2 p^{2}$ ). The proof is similar to the above, where if one function $h_{\mu}(\tau)$ is zero, then $h_{-\mu}(\tau)$ is also zero and this creates two nontrivial relations on all of the square classes. For the classes $v^{2} \bmod 2 p^{2}$ with only two elements, these relations are linearly independent and therefore the corresponding $h_{ \pm v}(\tau)$ are zero. Now there are $\varphi\left(2 p^{2}\right)$ relations among the $p$ elements in the 0 square class (also for the $p$ elements in the $p^{2}$ square class); however, only $p-1$ of these relations are linearly independent. For Jacobi forms of odd weight, each of the square classes $0, p^{2}$ has only $p-1$ elements (since $h_{0}, h_{p^{2}}=0$ ) and so the $p-1$ independent relations force all of the $h_{\mu}(\tau)$ to be zero.

If one of the $h_{\mu}(\tau)=0$ for $p \mid \mu\left(\mu \neq 0, p^{2}\right.$ if the weight is odd), then the Jacobi form is identically zero. This is because if $h_{\mu}(\tau)=0$ for $p \mid \mu$, then $h_{-\mu}(\tau)=0$ and the inversion formulas for these two give relations that imply $h_{v}(\tau)=0$ for all $(\nu, p)=1$ (instead of $h_{-\mu}$, use $h_{v}=(-1)^{k} h_{-v}$ if $\mu$ is 0 or $p^{2}$ and the weight is even). Now there are $\varphi\left(2 p^{2}\right)+2$ relations among the $p$ ( $p-1$ for odd weights) elements in the $0, p^{2}$ square classes of which $p$ are linearly independent and so the Jacobi form is identically zero.

Note that this result cannot be made much stronger. The reason is the operator $U_{l}$, where $l$ is a positive integer as defined in [3, page 41] that acts by $f(\tau, z) \mid U_{l}=$ $f(\tau, l z)$ and increases the index by $l^{2}$. A Jacobi form of index 1 has only two congruence 
classes of coefficients (in the $\xi$ ). Examples of such forms for even weights are explicitly constructed in [3] (there are no nonzero forms for odd weight). If one of these forms is lifted to level $p^{2}$ using $U_{p}$, then the image will only have nonzero $h_{\mu}(\tau)$ for congruence classes $\mu \bmod 2 m$, where $p$ divides $\mu$. In addition, by the above proposition, all of these components must be nonzero.

However, there is also a lowering operator or an inverse operator to $U_{l}$. Namely, if $F(\tau, z)$ is a Jacobi form of index $m$ such that $p^{2}$ divides $m$ and all of the associated $h_{\mu}(\tau)$ are zero unless $p$ divides $\mu$, then we can define an inverse of $U_{l}$ by

$$
F(\tau, z) \mid U_{l}^{-1}=\sum_{\mu \bmod \left(2 m / p^{2}\right)} g_{\mu}(\tau) \theta_{\mu, 2 m / p^{2}}(\tau, z),
$$

where

$$
g_{\mu}(\boldsymbol{\tau})=\sum_{\left\{a p \bmod 2 m \mid a \equiv \mu \bmod \left(2 m / p^{2}\right)\right\}} h_{a p}(\tau) .
$$

Then $F(\tau, z) \mid U_{l}^{-1}$ is a form of index $m /\left(p^{2}\right)$. To see this, we just check the vectorvalued modular form conditions. Namely, it is easy to see that

$$
g_{\mu}(\tau+1)=e\left(\frac{-\mu^{2}}{2 m / p^{2}}\right) g_{\mu}(\tau) .
$$

The inversion formula is only slightly more complicated and relies on the fact that the only nonzero components are the $h_{b p}$. Simply compute

$$
\begin{aligned}
g_{\mu}\left(\frac{-1}{\tau}\right) & =\sum_{\left\{a p \mid a \equiv \mu \bmod \left(2 m / p^{2}\right)\right\}} h_{a p}\left(\frac{-1}{\tau}\right) \\
& =\frac{(\tau)^{k-1 / 2}}{(\sqrt{2 m / i})} \sum_{\left\{a p \mid a \equiv \mu \bmod \left(2 m / p^{2}\right)\right\}} \sum_{\{v p \bmod 2 m\}} e_{2 m}(\operatorname{apvp}) h_{v p}(\tau) \\
& =\frac{(\tau)^{k-1 / 2}}{\sqrt{2 m / i}} \sum_{\left\{a p \mid a \equiv \mu \bmod \left(2 m / p^{2}\right)\right\}} \sum_{\left\{v \bmod \left(2 m / p^{2}\right)\right\}} \sum_{\left\{b p \mid b=v \bmod \left(2 m / p^{2}\right)\right\}} e_{2 m / p^{2}}(a b) h_{b p}(\tau) \\
& =(\tau)^{k-1 / 2} /\left(\sqrt{2 m / i p^{2}}\right) \sum_{v \bmod \left(2 m / p^{2}\right)} e_{2 m / p^{2}}(\mu \nu) g_{v}(\tau) .
\end{aligned}
$$

In particular, $F(\tau, z)\left|U_{l}\right| U_{l}^{-1}=l F(\tau, z)$. Therefore, all Jacobi forms with such a Fourier expansion are images of a form with lower index.

4. Jacobi forms on subgroups of the Jacobi group. In this section, we examine the Fourier expansions of Jacobi forms on subgroups of the Jacobi group and then investigate the relation to vector-valued modular forms for subgroups of $\mathrm{Sl}_{2}(\mathbb{Z})$. This method is applicable for any congruence subgroup. To begin, assume $f$ is a Jacobi form on $\Gamma(M) \ltimes(a)^{2}$, where $\Gamma(M)$ is some congruence subgroup of level $M$ and $(a)$ is 
the ideal generated by $a \in \mathbb{Z}, a \neq 0$. For a Jacobi form on this subgroup, $f(\tau+M, z)=$ $f(\tau, z+a)=f(\tau, z)$; so in this case, $f$ has a Fourier expansion of the form

$$
f(\tau, z)=\sum_{n} \sum_{r} c(n, r) q^{n / M} \xi^{r / a} .
$$

The analyticity condition on the second sum is that $4 m n / M-(r / a)^{2} \geq 0$. Following the classical argument (i.e., using the invariance with respect to the $\left.(a)^{2}\right)$, we find that $c(n, r)$ depends only on $r \bmod 2 m a^{2}$ and on $4 m n / M-(r / a)^{2}$. Replacing the variable $z$ by $a z$ (i.e., applying the operator $U_{a}$ as above), this becomes a Jacobi form of weight $k$ and index $a^{2} m$ on $\Gamma(M) \ltimes \mathbb{Z}^{2}$. So for the purposes of decomposing the Jacobi form, without loss of generality, we may consider just those forms on $\Gamma(M) \ltimes \mathbb{Z}^{2}$.

Now a Jacobi form $f(\tau, z)$ on $\Gamma(M) \ltimes \mathbb{Z}^{2}$ has a Fourier expansion

$$
f(\tau, z)=\sum_{n} \sum_{r} c(n, r) q^{n / M} \xi^{r}
$$

and as above, define $c_{\mu}\left(N=4 m n-M r^{2}\right)=c\left(\left(N+M r^{2}\right) / 4 m, r\right)($ where $r \equiv \mu \bmod 2 m$ and $c_{\mu}(N)=0$ if $\left.N \not \equiv-M \mu^{2} \bmod 2 m\right)$. Define the functions for $\mu \bmod 2 m$ :

$$
\begin{aligned}
h_{\mu}(\tau) & =\sum_{N \geq 0} c_{\mu}(N) q^{N / 4 m M}, \\
\theta_{\mu, m}(\tau, z) & =\sum_{\{r \in \mathbb{Z} \mid r \equiv \mu \bmod 2 m\}} q^{r^{2} / 4 m} \xi^{r} .
\end{aligned}
$$

These are exactly the same theta functions used in [3, page 58] and above. This leads to the decomposition

$$
\begin{aligned}
& \sum_{n} \sum_{r} c(n, r) q^{n / M} \xi^{r} \\
& \quad=\sum_{\mu \bmod 2 m} \sum_{r \equiv \mu \bmod 2 m} \sum_{\left\{N \geq 0 \mid N \equiv-M r^{2} \bmod 2 m\right\}} c\left(\frac{N+M r^{2}}{4 m}, r\right) q^{\left(N+M r^{2}\right) / 4 m M} \xi^{r} \\
& \quad=\sum_{\mu \bmod 2 m} h_{\mu}(\tau) \theta_{\mu, m}(\tau, z) .
\end{aligned}
$$

These theta functions $\theta_{\mu, m}(\boldsymbol{\tau}, z)$ satisfy

$$
\begin{gathered}
\theta_{\mu, m}(\tau+1, z)=e_{4 m}\left(\mu^{2}\right) \theta_{\mu, m}(\tau, z), \\
\theta_{\mu, m}\left(\frac{-1}{\tau}, \frac{z}{\tau}\right)=\sqrt{\tau / 2 m i} e^{2 \pi i m z^{2} / \tau} \sum_{\nu \bmod 2 m} e_{2 m}(-\mu \nu) \theta_{\nu, m}(\tau, z),
\end{gathered}
$$

but the Jacobi form only transforms with respect to $\Gamma(M)$; so we need to calculate how the $\theta_{\mu, m}$ transform with respect to these elements. To determine these formulas, it is sufficient to compute them on generators of the subgroup. In order to simplify the discussion for now, we consider the Hecke triangle and the $\Gamma_{0}(p)$ groups because they are generated by a small number of elements. To begin, let $\Gamma_{H}(M)$ be the Hecke triangle 
group generated by

$$
\left(\begin{array}{ll}
1 & 1 \\
0 & 1
\end{array}\right), \quad\left(\begin{array}{cc}
1 & 0 \\
M & 1
\end{array}\right)
$$

This group has infinite index in $\mathrm{Sl}_{2}(\mathbb{Z})$ for any $M>4$; however, this relatively small group is enough to restrict the possible Fourier expansions of a Jacobi form. Note that

$$
\left(\begin{array}{cc}
1 & 0 \\
M & 1
\end{array}\right)=\left(\begin{array}{cc}
0 & 1 \\
-1 & 0
\end{array}\right)\left(\begin{array}{cc}
1 & -M \\
0 & 1
\end{array}\right)\left(\begin{array}{cc}
0 & -1 \\
1 & 0
\end{array}\right)
$$

and we have the transformation for the translation matrix and so it remains to calculate the formula for the other generator. Using (4.5) and (4.6), the theta functions satisfy (using matrix notation for convenience)

$$
\begin{aligned}
\left(\theta_{\mu, m}\right. & \left.\left(\frac{\tau}{M \tau+1}, \frac{z}{M \tau+1}\right)\right)_{\mu \bmod 2 m} \\
= & -\frac{\sqrt{(M \tau+1)}}{2 m} e^{2 \pi i m M z^{2} /(M \tau+1)}\left(e_{2 m}(-\mu \nu)\right)_{\mu, v}^{-1} \\
& \times \operatorname{Diag}\left[e_{4 m}\left(-M \mu^{2}\right)\right]_{\mu}\left(e_{2 m}(-\mu \nu)\right)_{\mu, \nu}\left(\theta_{\nu, m}\right)_{\nu \bmod 2 m},
\end{aligned}
$$

where $\operatorname{Diag}[\cdot]$ is a diagonal matrix with the given entries and $\mu, v$ always run from $0,1, \ldots, 2 m-1$.

It is interesting to note at this point that if $2 \mathrm{~m} \mid \mathrm{M}$, this product of matrices becomes the identity and so each of the theta functions is a Jacobi form on $\Gamma_{H}(2 m) \ltimes \mathbb{Z}^{2}$. This may also be stated as the associated $h_{\mu}(\tau)$ are modular forms of weight $k-1 / 2$ on $\Gamma_{H}(2 m)$ (with a multiplier); or in representation-theoretic terms, this representation of $\mathrm{Sl}_{2}(\mathbb{Z})$ is reducible to one-dimensional representations on $\Gamma_{H}(2 m)$. Because of this fact, the only conditions on the $h_{\mu}(\tau)$ are that they be modular forms on $\Gamma_{H}(2 m)$ (with the appropriate multipliers). Furthermore, any such functions satisfying the above translation and inversion formulas may be put together with the theta functions to yield a Jacobi form on $\Gamma_{H}(2 m) \ltimes \mathbb{Z}^{2}$.

These transformation formulas for the theta functions can now be translated into transformation formulas for the $h_{\mu}$. Namely, since

$$
f(\tau, z)=\sum_{\mu \bmod 2 m} h_{\mu}(\tau) \theta_{\mu, m}(\tau, z)
$$

and $f$ is a Jacobi form on $\Gamma_{H}(M) \ltimes \mathbb{Z}^{2}$, each of the $h_{\mu}$ satisfies

$$
\begin{gathered}
h_{\mu}(\tau+1)=e_{4 m}\left(-\mu^{2}\right) h_{\mu}(\tau), \\
h_{\mu}\left(\frac{\tau}{M \tau+1}\right)=\frac{(M \tau+1)^{k-1 / 2}}{2 m} \sum_{\mu \bmod 2 m} a(\mu, \nu) h_{\nu}(\tau),
\end{gathered}
$$


where $a(\mu, v)$ is the $\mu, v$ entry in the inverse of the matrix in the theta function inversion. The matrix in this transformation of the $h_{\mu}(\tau)$ may be written as

$$
(a(\mu, v))_{\mu, v}=\left(\sum_{k=0}^{2 m-1} e_{4 m}\left(2(\mu-v) k+M k^{2}\right)\right)_{0 \leq \mu, v<2 m},
$$

where each entry is a form of a Gauss sum. These entries may be evaluated using standard techniques as in [1] and are explicitly calculated in [5]. A short calculation shows that the $\mu, v$ entry is zero unless $(M, 2 m) \mid(\mu-v)$, that is, unless $\mu \equiv v \bmod (M, 2 m)$. If $(2 m, M)=1$, then the $\mu, v$ entry is

$$
e\left(\frac{-M^{-1}}{2 m}(\mu-v)^{2}\right)\left(\frac{4 m}{M}\right)\left(1+i^{M}\right) \sqrt{m}
$$

where the inverse of $M$ is the inverse modulo $2 m$. If $(M, 2 m)=d>1$ (denote $M=d N$, $2 m=d n)$, then if $d \mid(\mu-v)$, the $\mu, v$ entry is

$$
a(\mu, v)= \begin{cases}\operatorname{de}\left(\frac{(-N)^{-1}}{2 n}\left(\frac{\mu-v}{d}\right)^{2}\right)\left(\frac{2 n}{N}\right)\left(1+i^{-N}\right) \sqrt{\frac{n}{2}} & \text { if } 2 \mid n, \\ d e\left(\frac{(-N)^{-1}}{8 n}\left(\frac{\mu-v}{d}\right)^{2}\right)\left(\frac{2 N}{n}\right) \sqrt{n} & \text { if } n \equiv 1 \bmod 4, \\ \operatorname{de}\left(\frac{(-N)^{-1}}{8 n}\left(\frac{\mu-v}{d}\right)^{2}\right)\left(\frac{2 N}{n}\right) i \sqrt{n} & \text { if } n \equiv 3 \bmod 4,\end{cases}
$$

where again $(-N)^{-1}$ is taken modulo $n$. These calculations lead to a statement (to [3, Theorem 5.1]) statement relating Jacobi forms and vector-valued modular forms on these Hecke groups.

THEOREM 4.1. The correspondence $f(\tau, z) \rightarrow\left(h_{\mu}(\tau)\right)_{\mu \bmod 2 m}$ gives an isomorphism between the spaces of Jacobi forms of weight $k$ and index $m$ on $\Gamma_{H}(M) \ltimes \mathbb{Z}^{2}$ and vectorvalued modular forms on $\Gamma_{H}(M)$ satisfying (4.11), (4.12) and bounded as $\operatorname{Im}(\tau) \rightarrow \infty$.

The above method is easily generalized to Jacobi forms on congruence subgroups by simply looking at the transformation of the theta functions on generators.

This theorem has an interesting consequence concerning how many of the vector components must be nonzero because of the condition that the entry of the matrix in (4.12) is zero unless $\mu=v \bmod (M, 2 m)$. This implies that the only functions $h_{v}(\tau)$, $0 \leq v<2 m$, involved in the transformation formula for $h_{\mu}(\tau /(M \tau+1))$ are those such that $\mu \equiv \nu \bmod (M, 2 m)$. Therefore, for a fixed congruence class $\mu$, as long as at least some of the $h_{v}$ for $v \equiv \pm \mu \bmod (M, 2 m)$ (there is still the relation that $\left.h_{v}=(-1)^{k} h_{-v}\right)$ are nonzero, all of the other components may be zero. Therefore, by increasing $(M, 2 m)$, the minimum number of nonzero components decreases.

COROLlary 4.2. If $f(\tau, z)$ is a Jacobi form of weight $k$ and index $m$ on $\Gamma^{J}$ with the decomposition (4.10) being satisfied and if $n$ divides $2 m$, then the function

$$
g_{\alpha, n}(\tau, z)=\sum_{v \equiv \pm \alpha \bmod n} h_{\nu}(\tau) \theta_{v, m}(\tau, z)
$$

is a Jacobi form on $\Gamma_{H}(n) \ltimes \mathbb{Z}^{2}$. 
Actually assuming the original form transforms on $\Gamma^{J}$ is overkill. All that is required is that it transform on a subgroup containing $\Gamma_{H}(n) \ltimes \mathbb{Z}^{2}$.

Thus we have that as the greatest common divisor of the level $M$ and the index $m$ increases, the minimum number of congruence classes (modulo $2 \mathrm{~m}$ ) of nonzero coefficients decreases. The first instance of a Jacobi form with only one nonzero $h_{\mu}(\tau)$ does not occur until level $m$ and then the only ones that occur have the form (assuming even weight) $h_{0}(\tau) \theta_{0, m}(\tau, z), h_{m}(\tau) \theta_{m, m}(\tau, z)$. At this level, we also have the first instance of only two of the $h_{\mu}(\tau)$ being nonzero (for index greater than 2). That is, given a Jacobi form on $\Gamma_{J}(\mathbb{Z})$, create the functions for $(\mu \bmod (m)) g_{\mu}(\tau, z)=$ $h_{\mu}(\tau) \theta_{\mu, m}(\tau, z)+h_{-\mu}(\tau) \theta_{-\mu, m}(\tau, z)$ which will be Jacobi forms on $\Gamma_{H}(m) \ltimes \mathbb{Z}^{2}$.

Now we consider Jacobi forms of index $m$ on the congruence subgroup $\Gamma_{0}(p) \ltimes \mathbb{Z}^{2}$, where $p$ is a prime that divides $m$. A convenient set of generators (see Frasch [4] and Rademacher [8]) for $\Gamma_{0}(p)$ is

$$
\begin{gathered}
\left(\begin{array}{ll}
1 & a \\
0 & 1
\end{array}\right)\left(\begin{array}{cc}
0 & -1 \\
1 & 0
\end{array}\right)\left(\begin{array}{cc}
1 & b \\
0 & 1
\end{array}\right)\left(\begin{array}{cc}
0 & 1 \\
-1 & 0
\end{array}\right)\left(\begin{array}{cc}
1 & a \\
0 & 1
\end{array}\right)\left(\begin{array}{cc}
0 & -1 \\
1 & 0
\end{array}\right) \\
=\left(\begin{array}{cc}
a-a(a b-1) & a b-1 \\
1-a b & b
\end{array}\right) \equiv V_{a, b},
\end{gathered}
$$

where $a$ runs through a set of congruence class representatives $p$, and $b$ is chosen so that $a b \equiv 1 \bmod p$. In order to make some of the calculations simpler, choose the representatives modulo $p$ so that each is relatively prime to $2 \mathrm{~m}$. In the case of $\Gamma_{0}(N)$ for general $N$, the generators are more complicated; see, for example, Chuman [2], and for the principal congruence groups of prime level, see Frasch [4]. Following the same method, as above, the transformation formulas for the $\theta_{\mu, m}(\tau, z)$ and the $h_{\mu}(\tau)$ are computed. The transformation matrix corresponding to the last three matrices in $V_{a, b}$ was calculated above and is

$$
\sqrt{m}\left(1+i^{-a}\right)\left(\frac{m}{-a}\right)\left(\exp \left(\frac{\pi i(v-\mu)^{2}\left(a^{-1}\right)}{2 m}\right)\right)_{0 \leq \mu, v<2 m}
$$

where $\left(a^{-1}\right)$ is the inverse modulo $2 \mathrm{~m}$. The transformation matrix corresponding to the first three elements of $V_{a, b}$ is

$$
\left(\exp \left(\frac{\pi i\left(-a \mu^{2}-b v^{2}+2 \mu \nu\right)}{2 m}\right)\right)_{0 \leq \mu, v<2 m}
$$

and thus their product is (neglecting the constant factors)

$$
\left(\sum_{k=0}^{2 m-1} \exp \left(\frac{\pi i}{2 m}\left(-a \mu^{2}+\left(a^{-1}\right) v^{2}+k^{2}\left[-b+\left(a^{-1}\right)\right]+2 k\left[\mu-\left(a^{-1}\right) v\right]\right)\right)\right)_{\mu, v}
$$

Now because $b$ was chosen to be equivalent to $\left(a^{-1}\right) \bmod 2 p$, the $k^{2}$ bracket is zero modulo $p$. So the sum is zero unless $p$ divides $\mu-\left(a^{-1}\right) v$. Therefore, the transformation of the $h_{\mu}(\tau)$ for the matrix $V_{a, b}$ depends only on the $h_{v}(\tau)$ for $b v$ equivalent to $\mu \bmod p$. 
In particular, if $p$ is a prime dividing $2 m$ and $p$ divides $\mu$, then $h_{\mu}(\tau)$ depends only on $h_{v}(\tau)$ for $p$ dividing $v$ for all of its transformations under $\Gamma_{0}(p)$. These considerations imply the following proposition (using the earlier notations).

Proposition 4.3. If $f(\tau, z)$ is a Jacobi form of weight $k$ and index $m$ on $\Gamma^{J}$ and $p$ is an odd prime dividing $m$, then $g_{0, p}(\tau, z), f(\tau, z)-g_{0, p}(\tau, z)$, and $f(\tau, z)$ (trivially) are Jacobi forms on $\Gamma_{0}(p) \ltimes \mathbb{Z}^{2}$. In addition, no other combinations of the $g_{\alpha, p}(\tau, z)$ are Jacobi forms on this subgroup.

So even on these subgroups, it is possible for more of the vector components, be zero but due to the transitive way the $V_{a, b}$ act on the vector components, there are only a limited number of ways.

5. Building Jacobi forms. In the preceding discussion, Jacobi forms were broken down into simpler forms on subgroups of the Jacobi group. This information allows one to reverse the process, that is, to begin with one (or more) of the $h_{\mu}(\tau)$ and create a Jacobi form from it. This is merely intended as an example to complement the above work; a more complete theory will appear in a forthcoming paper by the author.

To begin, assume that $h_{\mu}(\tau)$ is one of the associated vector-valued modular form components for a Jacobi form of weight $k$ and odd prime index $m=p$ for $\Gamma^{J}$, where $\mu$ is relatively prime to $2 \mathrm{~m}$. Define the natural slash operator for these vector-valued components by

$$
h_{\mu}(\tau) \mid\left(\begin{array}{ll}
a & b \\
c & d
\end{array}\right)=\chi\left(\begin{array}{ll}
a & b \\
c & d
\end{array}\right)^{-1}(c \tau+d)^{-k+1 / 2} h_{\mu}\left(\frac{a \tau+b}{c \tau+d}\right),
$$

where

$$
\left(h_{\mu}\left(\frac{a \tau+b}{c \tau+d}\right)\right)_{\mu \bmod 2 m}=\chi\left(\begin{array}{ll}
a & b \\
c & d
\end{array}\right)(c \tau+d)^{k-1 / 2} U\left(\begin{array}{ll}
a & b \\
c & d
\end{array}\right)\left(h_{v}(\tau)\right)_{v \bmod 2 m}
$$

$U\left(\begin{array}{ll}a & b \\ c & d\end{array}\right)$ is a unitary matrix, and $\chi$ is the multiplier. Using this slash operator and the earlier calculations, the other components of the vector-valued modular form are recovered (created):

$$
h_{a \mu}(\tau)=h_{\mu}(\tau) \mid V_{a, b},
$$

where $a$ runs from $1,3,5, \ldots, p-2$ and the $b$ 's are chosen so that $a b \equiv 1 \bmod 2 p$. Then using the relation $h_{v}(\tau)=(-1)^{k} h_{-v}(\tau)$, all of the vector components with odd subscripts are formed. Applying the slash operator with $\left(\begin{array}{ll}1 & 0 \\ p & 1\end{array}\right)$ to $h_{\mu}(\tau)$ gives the sum of $h_{\mu}(\tau)$ and $h_{\mu+p}(\tau)$ and so the vector components with even subscripts can now be recovered using the $V_{a, b}$ as above. This "new" vector function $\left(h_{\mu}(\tau)\right)_{1 \leq \mu \leq p-1}$ transforms as a vector-valued modular form on $\Gamma_{0}(p)$. In fact, this is the same Jacobi form as the one we began with except for the missing $h_{0}, h_{p}$ components. If the weight is odd, both of these are zero anyway, so we have recovered the original Jacobi form on $\Gamma^{J}$ (not just $\Gamma_{0}(p) \ltimes \mathbb{Z}^{2}$ ). If the weight is even, this is strictly a form on $\Gamma_{0}(p) \ltimes \mathbb{Z}^{2}$; however, we can add an $h_{0}(\tau)$ and $h_{p}(\tau)$ components by adding any pair of modular forms with 
the appropriate multipliers (as determined in the calculations for $V_{a, b}$ above) on $\Gamma_{0}(p)$. However, there is only one pair of these vector components which raises the form back to $\Gamma^{J}$. These components can be recovered through the series of operations

$$
h_{0}(\tau)=\left(h_{\mu}(\tau) \mid\left(\begin{array}{cc}
0 & -1 \\
1 & 0
\end{array}\right)-\sum_{\substack{v \neq 0, p \\
\nu \neq 0, p}} e_{2 m}(\mu \nu) h_{\nu}(\tau)\right)\left[1-i \mid\left(\begin{array}{ll}
1 & 1 \\
0 & 1
\end{array}\right)\right] .
$$

Similarly, $h_{p}(\tau)$ can be obtained by applying the same operator as above and replacing the $i$ with a 1 inside the brackets.

This last construction can also be done beginning with one of the $h_{\mu}(\tau)$, where $\mu$ is even $((\mu, p)=1)$ using almost the identical construction.

In the first part of this note, it was shown that a Jacobi form of odd prime index is entirely determined by any one of its associated vector-valued components and the above calculation showed how to generate the other components. There are still open questions. For example, the above construction of a Jacobi form was biased in that a priori, it was known that there was an associated Jacobi form. Given a function with some modular properties ((4.11), (4.12) for example), is there an attached Jacobi form? Is the above construction possible by starting with the $h_{0}$ component? How will this construction work for nonprime index, and how many of the components will be necessary to create a Jacobi form? In addition, what are the consequences for the associated half-integral weight modular forms as in Kohnen's papers [6, 7]?

\section{REFERENCES}

[1] B. C. Berndt, R. J. Evans, and K. S. Williams, Gauss and Jacobi Sums, Canadian Mathematical Society Series of Monographs and Advanced Texts, John Wiley \& Sons, New York, 1998.

[2] Y. Chuman, Generators and relations of $\Gamma_{0}(N)$, J. Math. Kyoto Univ. 13 (1973), 381-390.

[3] M. Eichler and D. Zagier, The Theory of Jacobi Forms, Progress in Mathematics, vol. 55, Birkhäuser Boston, Massachusetts, 1985.

[4] H. Frasch, Die Erzeugenden der Hauptkongruenzgruppen für Primzahlstufen, Math. Ann. 108 (1933), 229-252 (German).

[5] J. H. Hannay and M. V. Berry, Quantization of linear maps on a torus-Fresnel diffraction by a periodic grating, Phys. D 1 (1980), no. 3, 267-290.

[6] W. Kohnen, Modular forms of half-integral weight on $\Gamma_{0}(4)$, Math. Ann. 248 (1980), no. 3, 249-266.

[7] _ Newforms of half-integral weight, J. reine angew. Math. 333 (1982), 32-72.

[8] H. Rademacher, Über die Erzeugenden von Kongruenzuntergruppen der Modulgruppe, Abh. Math. Sem. Univ. Hamburg 7 (1929), 134-148 (German).

[9] N.-P. Skoruppa, Über den Zusammenhang zwischen Jacobiformen und Modulformen halbganzen Gewichts [On the Connection between Jacobi Forms and Modular Forms of Half-Integral Weight], Bonner Mathematische Schriften, vol. 159, Universität Bonn, Mathematisches Institut, Bonn, 1985.

Howard Skogman: Department of Mathematics, State University of New York at Brockport, Brockport, NY 14420, USA

E-mail address: hskogman@brockport. edu 


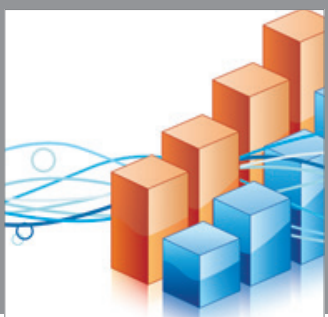

Advances in

Operations Research

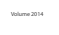

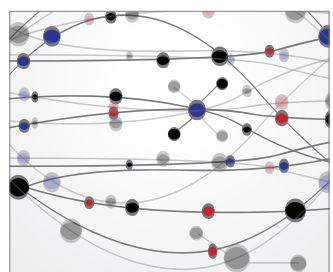

\section{The Scientific} World Journal
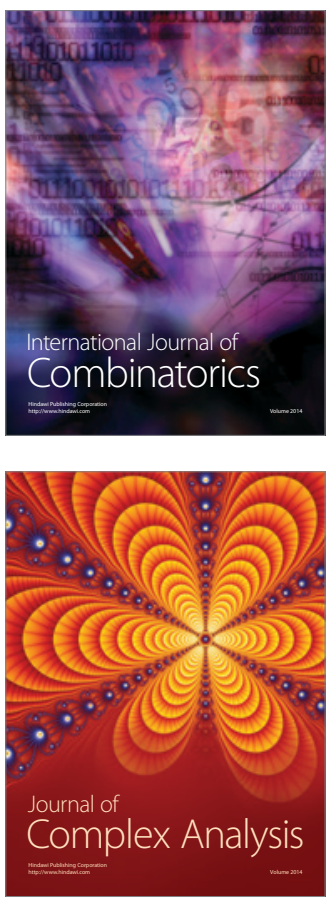

International Journal of

Mathematics and

Mathematical

Sciences
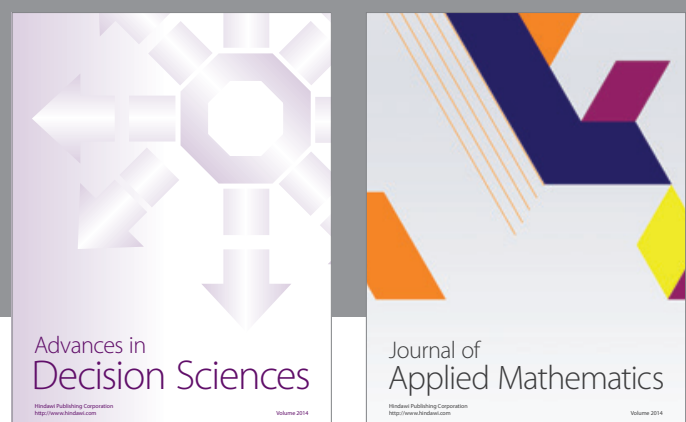

Journal of

Applied Mathematics
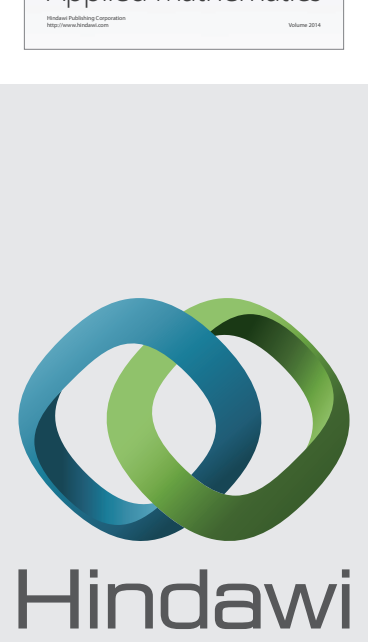

Submit your manuscripts at http://www.hindawi.com
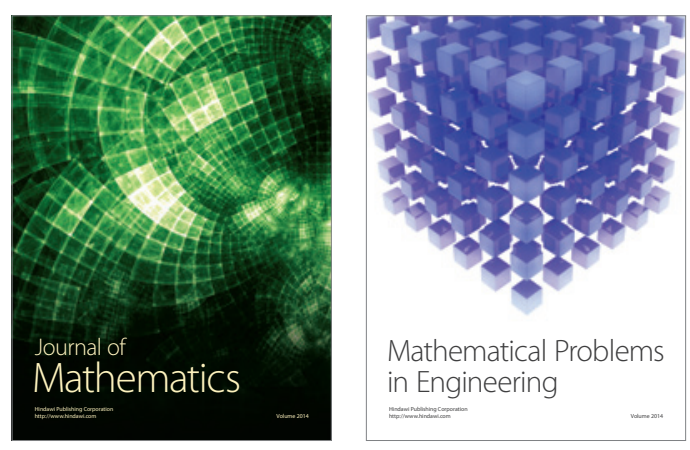

Mathematical Problems in Engineering
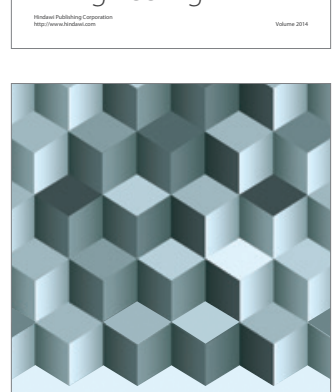

Journal of

Function Spaces
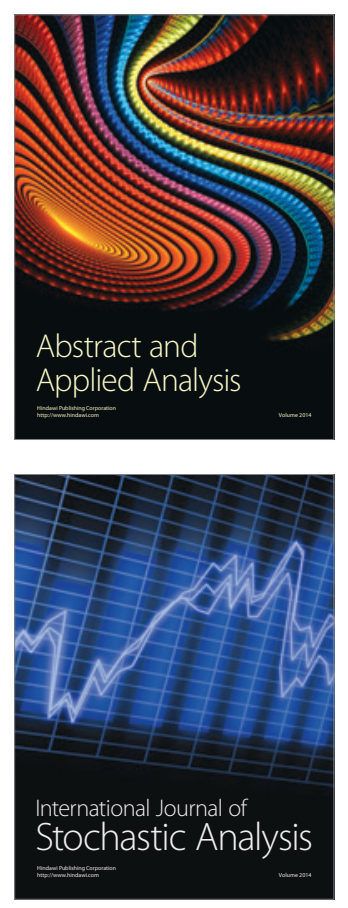

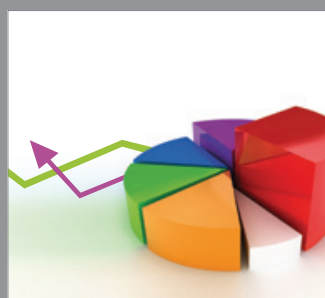

ournal of

Probability and Statistics

Promensencen
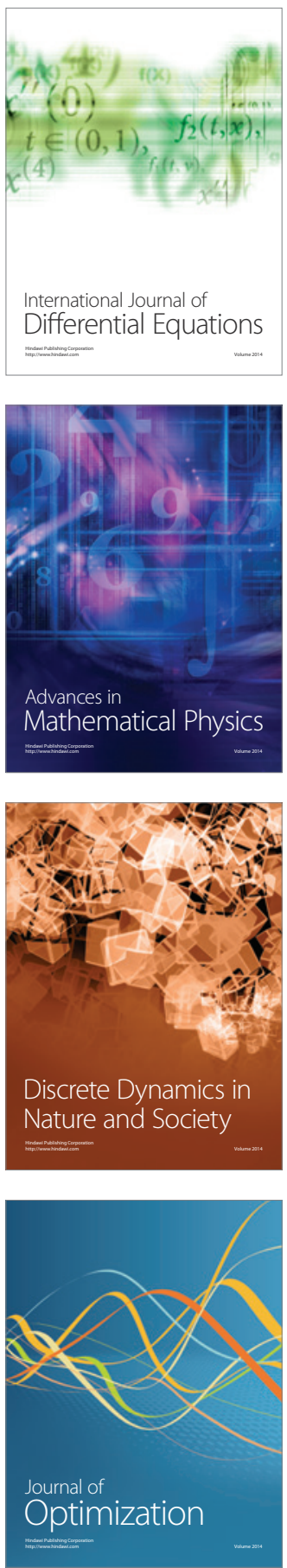